\title{
Simulation of Space Charge Effects in Electron Optical System Based on the Calculations of Current Density
}

\author{
Jiří Zelinka, Martin Oral and Tomáš Radlička
}

Institute of Scientific Instruments of the ASCR, v.v.i., Královopolská 147, 61264 Brno, Czech Republic

\begin{abstract}
We present a numerical method for iterative computation of electron optical systems influenced by space charge with an improved accuracy in the same calculation time. We replace the common algorithm for evaluating the space charge distribution with a new one based on the calculation of the current density distribution from an aberration polynomial. We introduce a re-meshing algorithm which adapts the mesh used for the field calculation by the finite element method in each iteration to the actual space charge distribution to keep it sufficiently fine in all areas with non-zero space charge.
\end{abstract}

\section{Introduction}

All electrons in the electron-optical system generally interact with each other. These repulsions affect the electron trajectories and the presence of the electrons itself alters the electrostatic field in the system. The significance of this effect grows with the total current in the system. It is not computationally feasible to simulate the interaction between each pair of electrons in the beam in most cases. The standard approximation uses the concept of space charge based on the collective effect. The space charge is a time invariant charge distribution corresponding to the actual current density distribution in the system. The space charge distribution generates a new electrostatic field in the system, leading to a new current density distribution, which allows performing a self-consistent computation [1].

The crucial part of the self-consistent algorithm, the evaluation of the space charge distribution, is commonly based on the particle-in-cell method [1] or the charge-tube method [2]. The particle in-cellmethod divides the simulated system to a large set of cells and determines the time the traced particles spend in each cell. It is necessary to have sufficiently small cells in the crossovers for good results. The formation of a hollow beam in the second iteration caused by a non-realistically high current density in the crossover was described in [1]. Other inaccuracies are caused by cells inside the wide parts of the beam not encountering any particles, which generates zero space charge. The problem with the accuracy in crossovers is improved by the charge tube method, which uses narrow tube cells around traced trajectories. On the other hand a huge number of trajectories has to be computed to eliminate the areas with no cells in the wide parts of the beam. Both methods have several empirical parameters, especially the charge-tube method is highly sensitive to the value of the tube diameter.

The calculation of the current density from the aberration polynomial is described in [3]. This method provides smooth results and it includes the effect of a non-zero source size and the energy distribution and can be used in all parts of the system where trajectory slopes are sufficiently small [4]. We implemented this approach to the space charge calculation and it led to more accurate results in the whole electron-optical system. The increased accuracy of the space charge distribution in each iteration improves convergence of the self-consistent iterative algorithm. Even our present non-optimized implementation retains about the same computation time as the two standard methods, even when a single iteration takes more time. 
The accuracy of the whole algorithm is strongly affected by the mesh used for the FEM field computations. A really fine mesh is necessary in those areas, where rapid changes in the field occur. These areas are determined by the fixed system geometry and the variable space charge distribution. It is difficult to construct a suitable mesh before starting the computation, especially if the number of elements should stay as low as possible for a reasonable computation speed. We solve this problem by a re-meshing algorithm based on [5], applied to the mesh in each iteration according to the actual current density distribution.

\section{Simulation algorithm}

We follow the standard self-consistent iterative computational scheme [1] in our space charge calculation method. The process starts with the computation of all fields not influenced by the space charge. After that the certain number if iterations is performed. In each iteration the actual current density distribution corresponding to the field from the previous iteration is evaluated. The mesh is refined according to the new current density distribution and the new electrostatic field is computed using the refined mesh. The computational scheme is shown in Figure 1.

We use the Galerkin method [6] with linear shape functions on a general triangular mesh in cylindrical coordinates for solving the field equations. The electrostatic part of the field is described by the standard Poisson equation for the electrostatic potential and the magnetic part is described by the equation for the magnetic flux function $\Psi(r, z)$. In the vicinity of optical axis with no materials we use the equation for the auxiliary potential function defined as $\Pi(r, z)=\Psi(r, z) / \pi r^{2}$ in coordinates $\left[r^{2}, z, \varphi\right]$, which provides near-axis $r^{2}$ correction [6]. Using the auxiliary potential function is advantageous due to the relation $B_{z}(0, z)=\Pi(0, z)$, so we do not have to perform the derivative on the axis to find the axial magnetic flux density. Details on the equations can be found in [7].

The aberration coefficients of a sufficient order in a fixed set of equidistantly distributed planes perpendicular to the optical axis are fitted using the standard least-squares method to the set of the traced trajectory points with initial conditions (particle positions, starting angles and energies) generated randomly with suitable distributions. These aberration coefficients are used to determine the positions of several billion particles in chosen planes perpendicular to the optical axis. It is important to choose relevant planes from the set of planes with known aberration coefficients, where current density is high or varies significantly, to prevent unnecessarily long computations. The current density distribution and its radial profile are constructed using the known particle positions for each chosen plane. The radial profiles are smoothed by Savitzky-Golay method [8] modified for non-equidistant abscissae and a quadratic spline is fit onto the resulting data. The fitted current density radial profiles are obtained in a few hundreds of the most relevant planes. These fits are used to construct a trilinear interpolant of the whole current density distribution which is very dense in the radial direction.

The re-meshing algorithm reduces each triangular element of the mesh to some maximal size by introducing new mesh points in the centers of the chosen edges. The criterion for the division is the maximum of the current density at the element centroid and at the centers of all its three edges. We use several predefined levels of this value corresponding to different maximal element sizes. This prevents abrupt changes in element sizes within small distances, which would cause inaccuracies in the FEM calculations. The regular structure in the reduced triangles is removed by a small random shift of the new points in the mesh at the end of the re-meshing process. The points of base mesh generated before the first iteration are held in fixed positions in all iterations, so that we can compare the results in them 
as a stopping criterion. As a result of the re-meshing process, there are no elements with the right hand side of the Poisson equation larger than some predefined maximum. The re-meshing in an iteration is based on the mesh generated in the previous one, which speeds up the process, because the same base mesh elements are not divided again in each iteration. The number of elements in the mesh grows with an increasing iteration number. Such a mesh in the third and higher iterations will typically have slightly more elements than the optimal one created by dividing the base mesh, but computational time of the remeshing together with the FEM field computation is significantly shorter.

We introduced the second order Anderson's mixing algorithm [9] to improve the convergence of whole self-consistent iterative algorithm. As the criterion of convergence we used the 2-norm of the differences in the electrostatic potential in two following iterations evaluated in the base mesh points. If the results of two following iterations are worse than the best result observed so far, the process is regarded as stagnating and the calculation is stopped too.

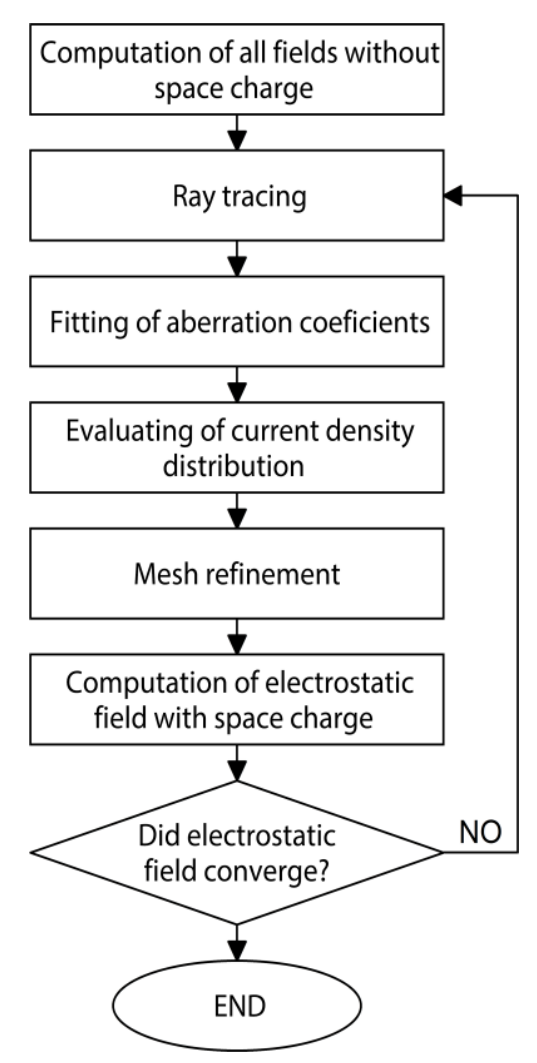

Figure 1. The simplified algorithm diagram based on the standard iterative self-consistent method used for space charge computations [1].
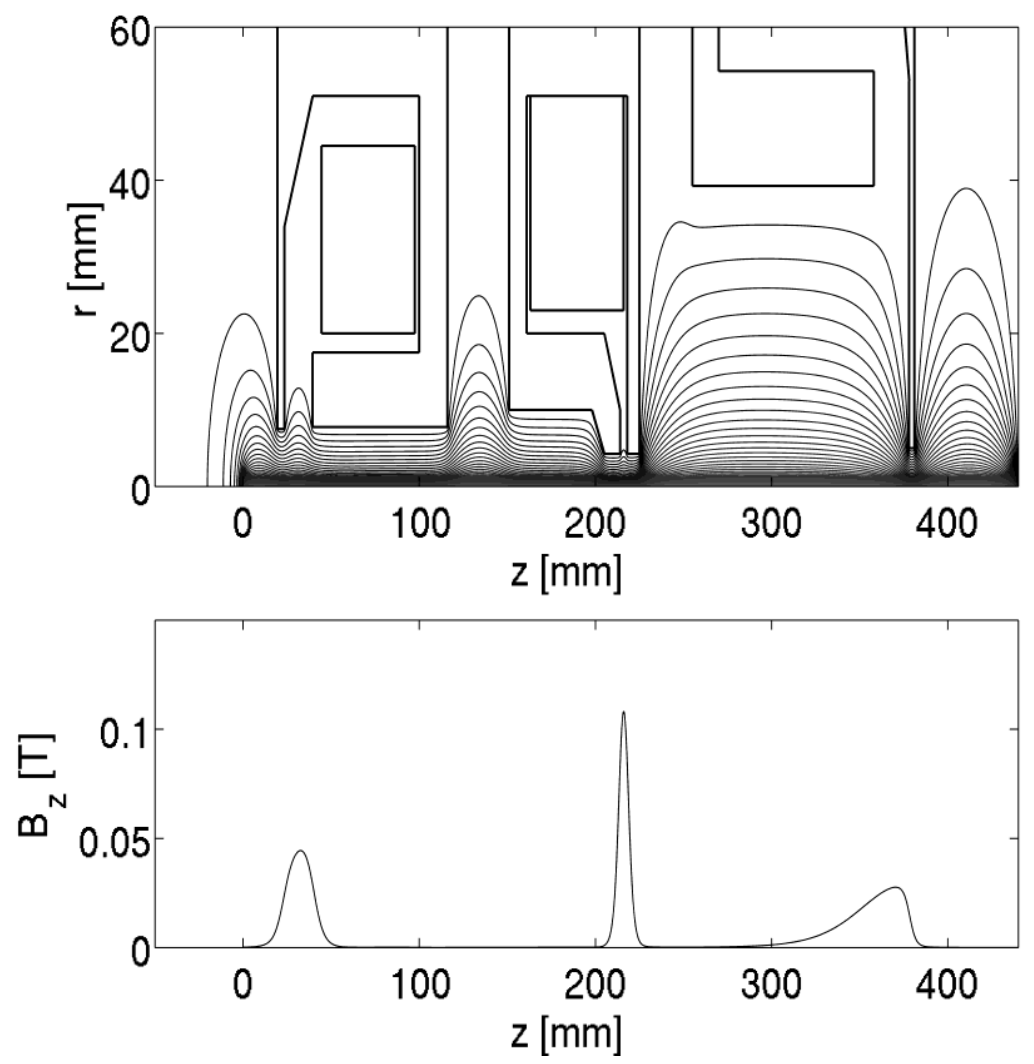

Figure 2. The upper image shows the geometry of the simulated system with the electrostatic field in the second iteration based on the trajectories traced only in the magnetic field and without space charge. The lower image shows the axial z-component of magnetic flux density not influenced by space charge.

\section{Algorithm testing}

We implemented the proposed algorithm in MATLAB. The final FEM system of linear equations with a sparse matrix was solved by the MATLAB bicgstab procedure with a Jacobi preconditioner [10]. The 
electrostatic field from the previous iteration was used as the initial guess to speed up the computation. The trajectories were traced using MATLAB Runge-Kutta ode45 solver calculating the standard relativistic trajectory equation [7]. The electrostatic field influenced by the space charge was interpolated by the ZRP method [11] adapted for the Poisson equation. The interpolation of the magnetic flux density vector components was based on the seventh-order expansion of the scalar magnetic potential [7].

We tested our algorithm on the design of the electron lithograph ELG BS 600 consisting of three magnetic lenses, showed in Figure 2, in an imaging regime with two crossovers. The starting plane corresponds to the crossover of the first condenser lens, where we used Gaussian distributions of the initial positions with $\sigma=0.003 \mathrm{~mm}$ and of the energy deviations with $\sigma=1.5 \mathrm{eV}$ and uniformly distributed trajectory slopes of up to 0.005 . The nominal energy was $15 \mathrm{keV}$ and the total current in the system was $1 \mu \mathrm{A}$. The virtual source was considered in the starting plane. The small beam diameter in the starting plane generates an undesired sharp peak in the space charge distribution which was not taken into account in our model of the virtual source. To fulfill the condition of the constant current in the entire system, in the vicinity of the staring plane we set a constant space charge distribution according to some plane a few millimeters behind the starting plane. The Dirichlet boundary condition on the left border was located a few centimeters from the starting plane, which slightly enlarges the mesh, but it significantly improves the electrostatic field in the vicinity of the starting plane. The base mesh generated by the software Gmsh 2.8.4 [12] had 2.35 million elements. The number of the elements after the first refinement was 3.24 million and went up to 5.28 million in the last iteration.

The calculation time of one iteration was around 15 hours on a $4 \mathrm{GHz} 8$-core AMD-FX computer, but the code is not well optimized yet. The stopping tolerance was set to $1 \mu \mathrm{V}$. We performed 8 iterations until the stopping criteria were fulfilled; the best result was observed in the sixth iteration with the 2norm of the potential difference of $0.98 \mathrm{mV}$. We checked the accuracy of proposed method of evaluating current density distribution after the computation. We fitted the aberration polynomial of the fifth order to the set of 2500 trajectories and the residual standard deviation was several nanometers, which is demonstrated in Figure 3.
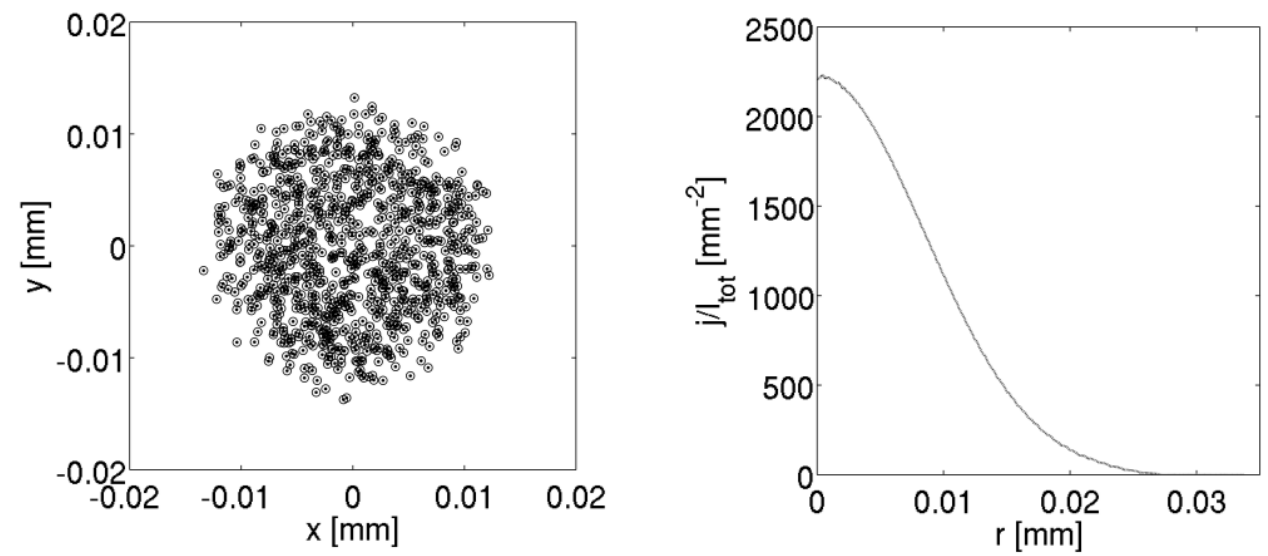

Figure 3. The plot on the left shows a comparison of the ray-traced positions in the given plane (full dots) and the positions calculated by evaluating the aberration polynomial of the fifth order (circles). The plot on the right shows the current density distribution radial profile obtained using the same aberration polynomial in the same plane. The fitted quadratic spline is drawn by dashed gray line nearly overlaps the data after Savitzky-Golay smoothing represented by full black line. 


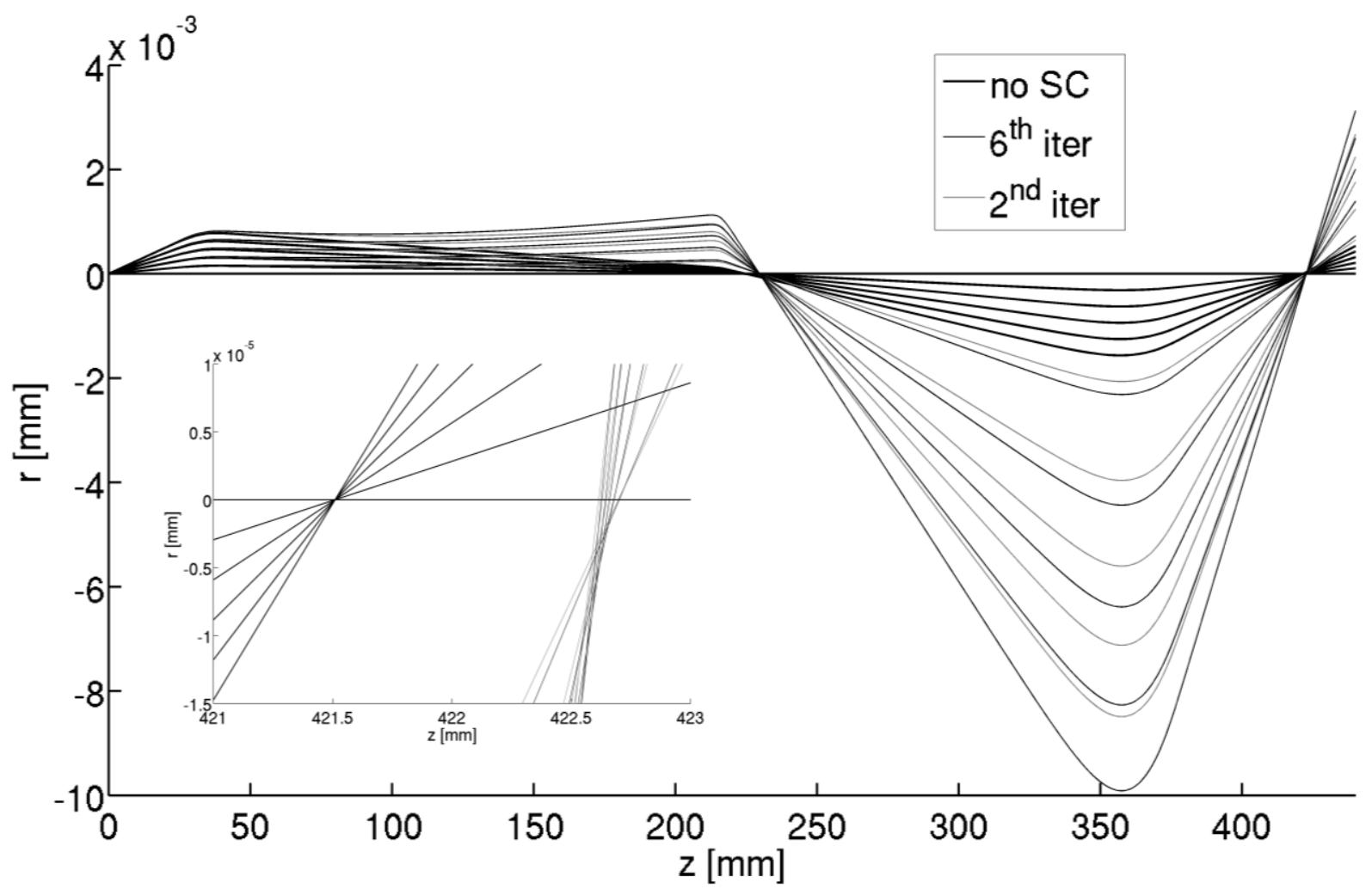

Figure 4. Trajectories starting on the optical axis with the nominal energy under different equidistant slopes, in the rotating coordinates. The inset graph shows the vicinity of the second crossover.
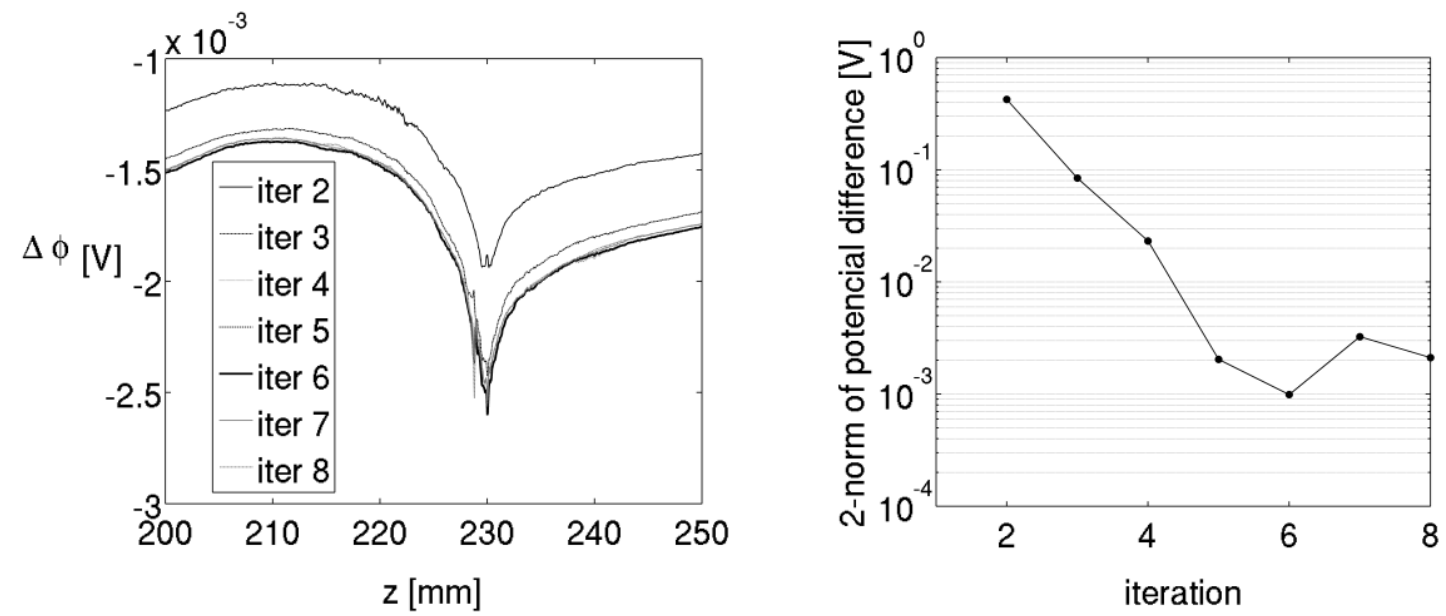

Figure 5. The graph on the left shows the evolution of the electrostatic potential near the first crossover in the base mesh axial points in different iterations. The thick black line represents the result for the sixth iteration, from which the results were chosen. The changes following this iteration are small. The graph on the right shows the convergence of the algorithm. It is possible that algorithm would converge to slightly better results, but in a much longer computation time.

We made the comparison of the results without the space charge and those after six iterations. In Figure 4 it is obvious that trajectories with the same initial conditions change significantly. The diverging 
action of the space charge is clearly visible especially in front of the first crossover. We made a comparison of the crossover positions and the diameters. The presented beam diameters correspond to the diameter containing $50 \%$ of the total beam current. The positions of crossovers were determined by local minima in the beam size. The changes in these two quantities are presented in Table 1 , both crossover sizes decrease in the presence of space charge. This fact is illustrated by the imaging parameters of Gaussian planes presented in Table 1 too.

\begin{tabular}{|c|c|c|c|c|c|c|c|c|c|c|}
\cline { 2 - 11 } \multicolumn{1}{c|}{} & \multicolumn{2}{|c|}{$1^{\text {st }}$ Gaussian plane } & \multicolumn{2}{c|}{$1^{\text {st }}$ crossover } & \multicolumn{2}{c|}{$2^{\text {nd }}$ Gaussian plane } & \multicolumn{2}{c|}{$2^{\text {nd }}$ crossover } \\
\cline { 2 - 11 } & position [mm] & $\mathrm{M}$ & $\mathrm{m}$ & position $[\mathrm{mm}]$ & size $[\mu \mathrm{m}]$ & position $[\mathrm{mm}]$ & $\mathrm{M}$ & $\mathrm{m}$ & position $[\mathrm{mm}]$ & size $[\mu \mathrm{m}]$ \\
\hline no SC & 224,95 & $-1,953$ & $-0,5124$ & 229,75 & 1,755 & 421,5 & 0,873 & 1,156 & 422,5 & 0,823 \\
\hline SC & 229,15 & $-0,440$ & $-2,2413$ & 229,85 & 1,499 & 422,45 & 0,222 & 4,910 & 422,55 & 0,704 \\
\hline
\end{tabular}

Table 1. Comparison of the properties in the planes of least confusion and in the Gaussian image planes in the system with and without space charge.

\section{Conclusions}

The proposed algorithm converges in the order of magnitude of tens of hours on a $4 \mathrm{GHz} 8$-core AMDFX computer. It was tested on an electron-optical system with two crossovers. The shift of the second image is only several hundredths of a millimeter along the optical axis. The crossover diameters were reduced by a few tenths of a micrometer as a result of the axial space charge distribution. The algorithm can be used in those parts of the electron-optical system, where the trajectories can be fitted by the aberration polynomial. In the vicinity of the cathode the aberration polynomial cannot be used in the standard form. Further work will be focused on the determination of the initial conditions in the starting plane of the system and with the simulation in the vicinity of cathode [13].

\section{References:}

[1] P Jánský et al, Vacuum 84 (2009), p. 357.

[2] F Read, Proceedings of SPIE, CPO IV (1999) p. 184.

[3] M Oral and B Lencová, Nucl. Instrum. Meth. B 645 (2011), p. 130.

[4] M Oral and B Lencová, Ultramicroscopy 109 (2009), p. 1365.

[5] MA Padrón et al, Math. Comput. Simul. 75 (2007), p. 251.

[6] A Khursheed in "The Finite Element Method in Charged Particle Optics", (Kluwer Academic Publishers, Boston) p. 84.

[7] PW Hawkes and E Kasper in "Principles of Electron Optics", (Academic Press, London) p.27.

[8] A Savitzky and JE Golay, Anal. Chem 36 (1964), p. 1627.

[9] DD Johnson, Phys. Rev. B 38 (1988), p. 12807.

[10] R Barrett et al in "Templates for the Solution of Linear Systems: Building Blocks for Iterative Methods", (SIAM, Philadelphia) p. 35.

[11] J Chmelík and JE Barth, Proceedings of SPIE, CPO (1993) p. 133

[12] C Geuzaine and JF Remacle, Int. J. Numer. Meth. Eng. 79 (2009), p. 1309.

[13] The authors acknowledge the support from Ministry of Education, Youth and Sports of the Czech Republic (LO1212) together with the European commission (ALISI No. CZ.1.05/2.1.00/01.0017). 